\title{
Weak matrix elements for CP violation *
}

\author{
T. Bhattacharya ${ }^{\mathrm{a}}$, G.T. Fleming ${ }^{\mathrm{b}}$, G. Kilcup ${ }^{\mathrm{b}}$, R. Gupta ${ }^{\mathrm{a}}$, W. Lee ${ }^{\mathrm{a}}$ and S. Sharpe ${ }^{\mathrm{c}}$ \\ a MS-B285, Group T-8, Los Alamos National Lab, Los Alamos, New Mexico 87545, USA \\ bepartment of Physics, Ohio State University, Columbus, OH 43210, USA \\ ${ }^{\mathrm{c}}$ Department of Physics, University of Washington, Seattle, WA 98195, USA
}

We present preliminary results of matrix elements of four fermion operators relevant to the determination of $\epsilon$ and $\epsilon^{\prime} / \epsilon$ using staggered fermions.

\section{INTRODUCTION}

To calculate the matrix elements relevant to $\mathrm{CP}$ violation in Kaon decays it is important to use a lattice formulation which preserves (some) chiral symmetry. In the case of $B_{K}, B_{7}^{(3 / 2)}$, and $B_{8}^{(3 / 2)}$, the absence of chiral symmetry leads to mixing with wrong chirality operators, which in turn leads to large discretization errors. The problem is far more severe for $B_{6}^{(1 / 2)}$ due to mixing with lower dimension operators. Two lattice formulations that respect at least part of the continuum chiral transformations and hold promise for these calculations are domain wall/overlap fermions and staggered fermions. Each has its advantages and disadvantages. Renormalization of operators in the domain wall/overlap formulation is small enough that 1-loop calculations may be adequate, but the numerical simulations are $\sim 100$ times more costly. Staggered simulations are very efficient, but the 1-loop renormalization constants for the simplest lattice transcription of operators are very large. The goal of this project is to find an improved staggered formulation for which perturbation theory is well-behaved.

Here we present preliminary estimates of $B_{K}$, $B_{7}^{(3 / 2)}, B_{8}^{(3 / 2)}$, and $B_{6}^{(1 / 2)}$ using 140 quenched lattices $\left(16^{3} \times 64\right)$ at $\beta=6.0$. This numerical simulation is being done on the QCDSP supercomputer at Columbia University. To facilitate chiral extrapolations, we have used four values of quark

\footnotetext{
*Poster presented by W. Lee ${ }^{\mathrm{a}}$, This work has been sup-
} ported by DOE. mass: $a m_{q}=0.01,0.02,0.03,0.04$. The results are for gauge invariant staggered operators that lie in a $2^{4}$ hypercube for which the 1-loop renormalization constants are now known [1].

2. $B_{K}$

We have used the calculation of $B_{K}$ as a test of our programs. The estimates shown in Figure 11 agree with previous calculations [2 -4] and it is worth mentioning that the 1-loop calculations done independently in Ref. [1] reproduce the results given in Ref. [5]. The figure shows a fit using the form suggested by chiral perturbation theory, $c_{0}+c_{1}\left(a M_{K}\right)^{2}+c_{2}\left(a M_{K}\right)^{2} \ln \left(a M_{K}\right)^{2}$, with $c_{0}=0.55(8), c_{1}=0.06(25)$, and $c_{2}=-0.60(38)$. Physical kaons correspond to $\left(a M_{K}\right)^{2} \approx 0.06$.

\section{3. $B_{7}^{(3 / 2)}$ and $B_{8}^{(3 / 2)}$}

In Figures 2 and 3, we compare the tree level and one-loop results for $B_{7}^{(3 / 2)}$ and $B_{8}^{(3 / 2)}$ (defined in [2]). The 1-loop tadpole improved renormalization constants for the gauge invariant operators were recently calculated in [1]. We match to the continuum NDR scheme at $q^{*}=\mu=$ $\pi / a$. The dominant contribution to $\left\langle O_{8}^{(3 / 2)}\right\rangle$ comes from 2-color trace staggered operator $[P \times$ $P][P \times P]_{I I}$, which also dominates the vacuum saturation contribution. Thus, even though the renormalization constants are large, there is a close cancellation and the $B$ parameter receives $\mathrm{a} \lesssim 10 \%$ 1-loop correction. On the other hand 


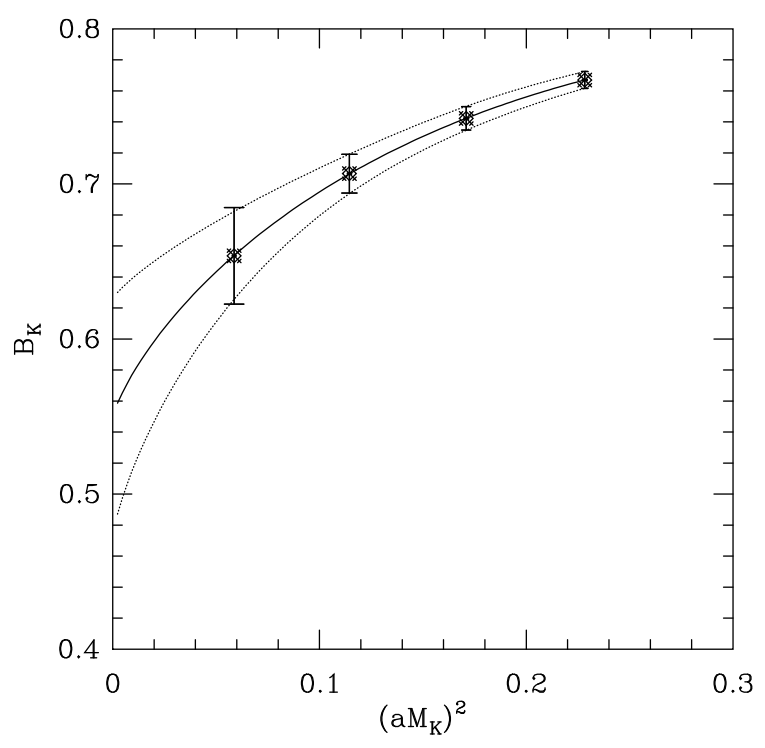

Figure 1. $B_{K}(\mu=\pi / a, N D R)$.

$\left\langle O_{7}^{(3 / 2)}\right\rangle$ is dominated by the 1 -color contraction, and the renormalization constants do not cancel. Consequently, even assuming $q^{*}=\pi / a, 1$-loop perturbation theory is unreliable.

These results can be compared against previous calculations done using gauge non-invariant Landau gauge operators in Ref. [2]. The authors of Ref. [2] found significant systematic differences between results obtained using smeared and unsmeared operators. Choosing the same value of $\beta$ and $a m_{q}$, we find that our results lie in between.

\section{4. $B_{6}^{(1 / 2)}$}

Accurate estimates of $\epsilon^{\prime} / \epsilon$ require measurements of matrix elements of QCD and electromagnetic penguin operators, $\left\langle O_{6}^{(1 / 2)}\right\rangle$ and $\left\langle O_{8}^{(3 / 2)}\right\rangle$ respectively, in the $K \rightarrow \pi \pi$ transition. Since these two contribute with opposite sign, leading to a significant cancellation, both need to be measured precisely in order to test whether the Standard Model explains the observed size of $\operatorname{Re}\left(\epsilon^{\prime} / \epsilon\right)$.

Direct calculations of the $K \rightarrow \pi \pi$ amplitudes on the lattice are difficult $\mid 6]$. The simplest approach has been to assume chiral pertur-

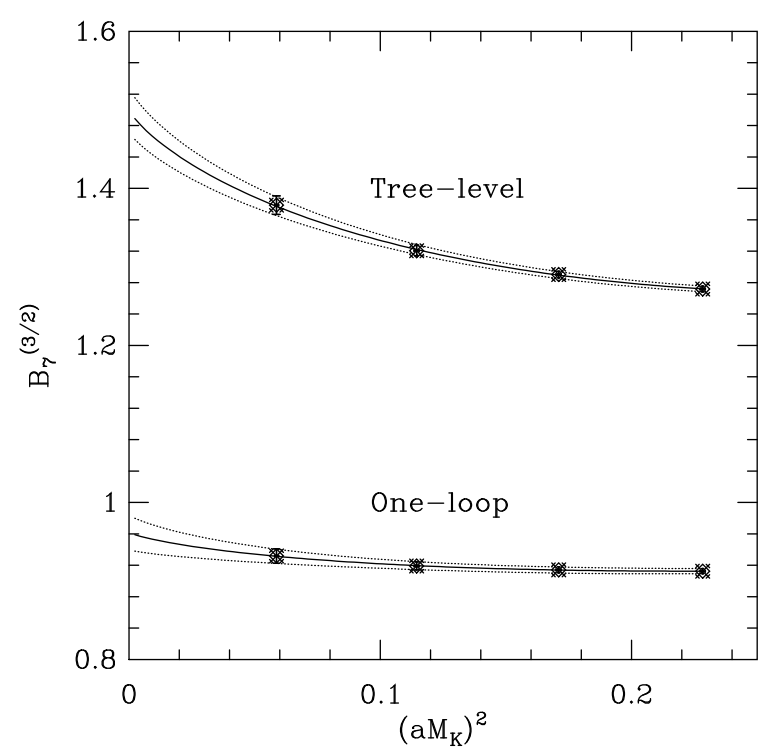

Figure 2. $B_{7}^{(3 / 2)}(\mu=\pi / a, N D R)$.

bation theory provides accurate relations between $K \rightarrow \pi \pi, K \rightarrow \pi$ and $K \rightarrow 0$ amplitudes [7]. In this method, which we use, the operator $O_{6}^{(1 / 2)}$ has three types of contractions: eight, eye, and subtraction (to remove mixing with lower dimension operators) [幽]. Schematically,

$$
\begin{aligned}
B_{6}^{(1 / 2)} & =\frac{\left\langle K^{+}\left|O_{6}^{1 / 2}\right| \pi^{+}\right\rangle}{\text {Vac. Sat. }} \\
O_{6}^{1 / 2} & =O_{6}^{1 / 2}(\text { Eight })+O_{6}^{1 / 2}(\text { Eye })+O_{6}^{1 / 2}(\text { Sub })
\end{aligned}
$$

In order to restrict the subtraction term to the single dimension four operator $O_{\text {sub }} \equiv\left(m_{d}-\right.$ $\left.m_{s}\right) \bar{s} \gamma_{5} d+\left(m_{d}+m_{s}\right) \bar{s} d$, we need to work with degenerate $s$ and $d$ quarks. For $m_{s}=m_{d}$ we can determine the coefficient $\alpha$ of $O_{\text {sub }}$ by calculating the derivative of the $K \rightarrow 0$ amplitude with respect to the strange quark mass. This derivative introduces two types of diagrams: (i) each strange quark propagator is replaced by its derivative, (ii) a disconnected diagram that arises from the differentiation of the fermion determinant. In principle, both terms can be estimated in the quenched theory. However, the disconnected contribution vanishes in the limit of $m_{s}=m_{d}$. Hence, our calculation of $\alpha$ does not include the disconnected contributions and is thus directly 


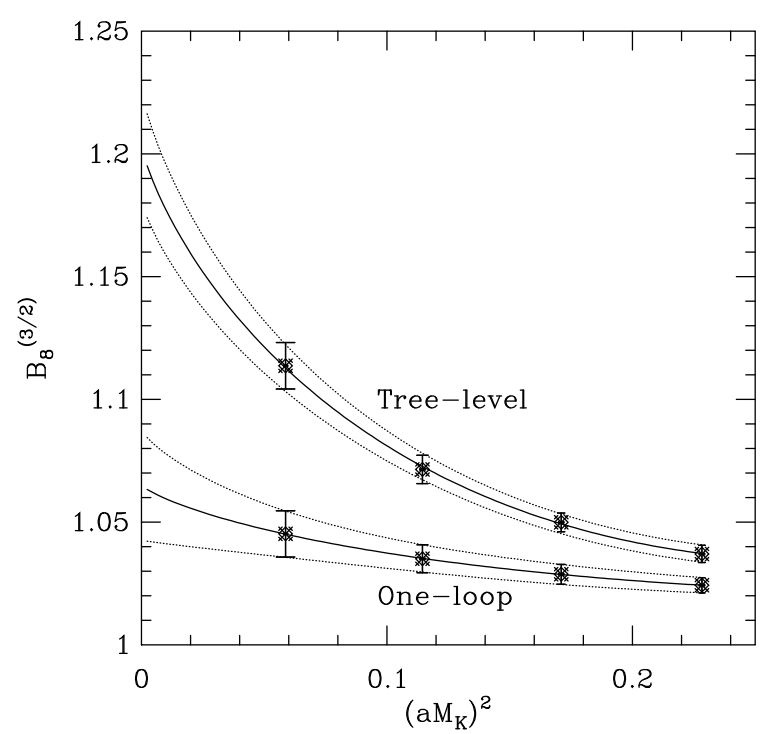

Figure 3. $B_{8}^{(3 / 2)}(\mu=\pi / a, N D R)$.

comparable to those of Refs. [4, 8,9], which do not discuss such contributions. The results are shown in Figure 4, labeled as "standard". The details will be presented in Ref. [10].

Recently, Golterman and Pallante have pointed out a subtlety associated with the quenched approximation using (partially) quenched chiral perturbation theory [11. They show that the usual quenched operators lead to matrix elements which have a different chiral expansion from that in the continuum. They propose an alternative quenched operator which does not have these problems. In practice this amounts to dropping certain contractions in the "eye" and "subtraction" diagrams.

We demonstrate in Figure 1 the difference in $B_{6}$ using the standard operator and that suggested by Golterman and Pallante. The 1-loop renormalization constants in the NDR scheme are from Ref. [1] and [12]. Our calculations show that the Golterman-Pallante operator enhances $B_{6}$ by almost a factor of two, which in turn would significantly increase the value of $\epsilon^{\prime} / \epsilon$ compared to results given in [4,9]. Thus the quenched approximation remains the most significant drawback of such calculations, which we hope to address in the future.

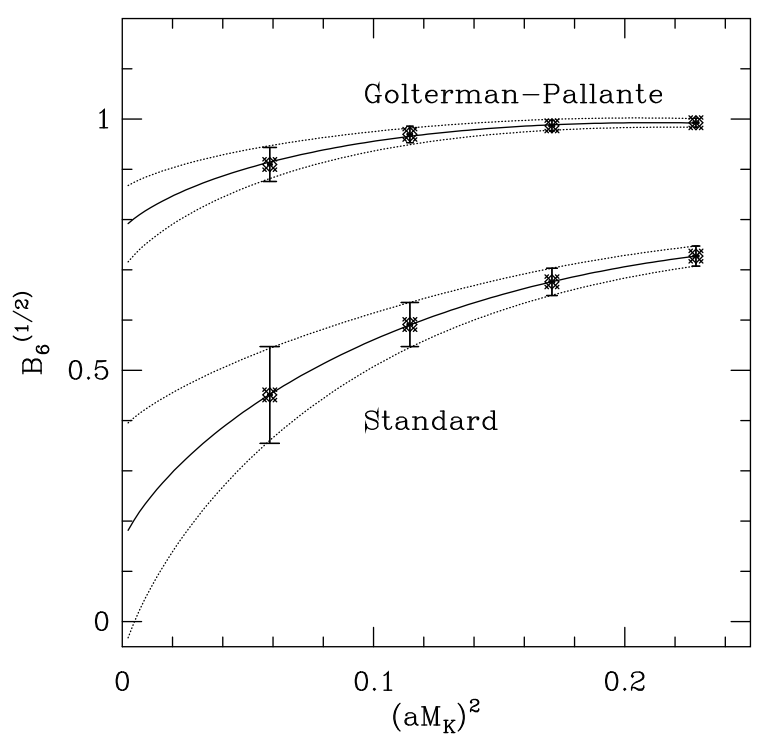

Figure $4 . B_{6}^{(1 / 2)}(\mu=\pi / a, N D R)$ using the standard operator and Golterman-Pallante operator.

We thank N. Christ, G. Liu, R. Mawhinney and $\mathrm{L}$. Wu for their support of this project and assistance with simulations on the Columbia supercompter, QCDSP, on which this calculation was performed.

\section{REFERENCES}

1. W. Lee, Phys. Rev. D64 (2001) 054505.

2. G. Kilcup, R. Gupta, and S. Sharpe, Phys. Rev. D57 (1998) 1654.

3. S. Aoki, et. al., Phys. Rev. Lett. 80 (1998) 5271.

4. D. Pekurovsky and G. Kilcup, Phys. Rev. D64 (2001) 074502.

5. N. Ishizuka and Y. Shizawa, Phys. Rev. D49 (1994) 3918.

6. C. Lin, et. al., hep-lat/0104006.

7. C. Bernard, et. al., Phys. Rev. D32 (1985) 2343.

8. T. Blum, et. al., hep-lat/0110075.

9. J. Noaki, et. al., hep-lat/0108013.

10. W. Lee, et. al., in preparation.

11. M. Golterman, et. al., hep-lat/0108010.

12. S. Sharpe and A. Patel, Nucl. Phys. B417 (1994) 307. 